
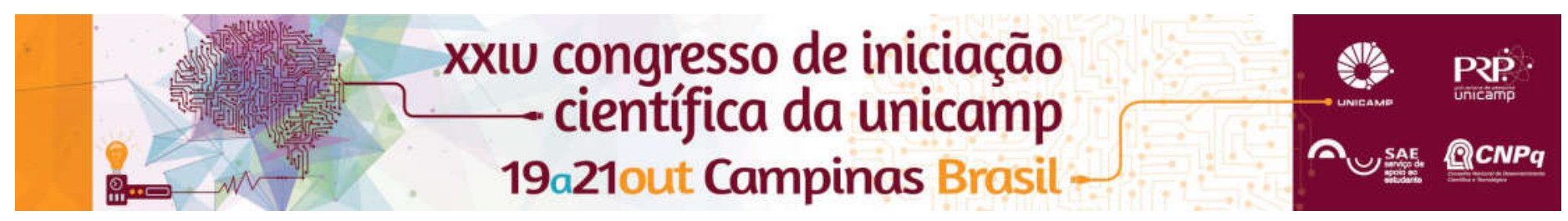

\title{
Study of Residential Sensing with Internet Of Things
}

\section{Thallyson Paulino*, Luis Avila}

\begin{abstract}
Smart homes use automatic methods for control and management of the environments. This research aims to integrate these methods with Internet of things.
\end{abstract}

\section{Key words: \\ Home Automation, Internet Of Things, Sensors.}

\section{Introduction}

The relationship between objects and devices through the concept of internet of things can be defined as the intersection between the physical, social, real time and programable internet and can be applied in different environments. On the other hand, the smart homes work with the control and use of the energy, management of water, monitoring of the temperature and humidity, as well as, the control of the motion. This research integrate a smart home system with a wireless communications set, sensors, protocols of the internet of things, cloud computing, embedded systems and data mining in a simulation environment. This simulation looks for a proof of concept of smart home integrated with internet of things.

\section{Results and Discussion}

The simulation of a home automation system can be performed according with an application diagram, shown in Image 1. The main components are a microcontroller (in this research, the Intel Galileo is used), general sensors (temperature, motion) and a relay module. These components should be tested individually and together, according to a sketch created on a Arduino Software. This software works together a web server, controling and monitoring the home automation system through the internet.

The microcontroller Intel Galileo is connected to an Ethernet Connection and a Micro SD onboard, as shown in Image 1. Sensors and electronic components are also connected to Galileo and, through a Sketch created on Software Arduino, is possible to access Putty, an application who makes the interface between the microcontroller and the internet. Using this application is possible to access the embedded system Linux installed on Galileo's Micro SD. This embedded system is the Yocto platform that offers compilers like Node and Phyton and works with JavaScript codes, creating a server. The server works together with the Arduino Software Sketch sending and receiving data to/from the sensors and components connected on Galileo.

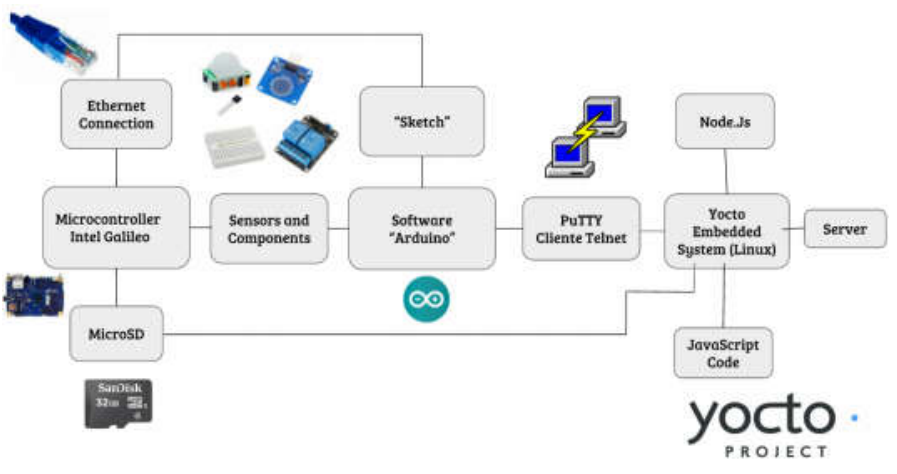

Image 1. Application Diagram.

The system developed in this research perform the control of the telectric devices (such as: a lamp, a fan, a coffee machine) through the internet. A webpage can be used to monitor the status of these devices. Commands like "Onrelay1", "Offrelay1", "Onrelay2", "Offrelay2" can be sent to the webpage, acting directly to the electric devices connected on plug and on the Galileo, turning on or turning off them.

\section{Conclusions}

In this research, it was possible to simulate a home automation system that controls electronic devices using the concepts of the Internet of Things.

\section{Acknowledgement}

The authors thank to the Programa Institucial de Bolsas de Iniciação em Desenvolvimento Tecnológico e Inovação - PIBITI/CNPq for the financial support.

\footnotetext{
${ }^{1}$ Ilker Korkmaz, Senem Kumova Metin, Alper Gurek, Caner Gur, Cagri Gurakin, Mustafa Akdeniz, "A cloud based and Android supported scalable home automation system", Computers \& Electrical Engineering, 43, 112-128, 2015.

${ }^{2}$ Manoel Carlos Ramon, " Intel Galileo and Intel Galileo Gen 2 - API Features and Arduino Projects for Linux Programmers", Copyright 2014 by Apress Media, LLC, all rights reserved.
} 